\title{
Positive modulation of a neutral declarative memory by a threatening social event
}

\author{
Rodrigo S. Fernández ${ }^{\text {a }}$, Luz Bavassi ${ }^{\mathrm{a}, \mathrm{b}}$, Jorge Campos $^{\mathrm{c}}$, Ricardo F. Allegri ${ }^{\mathrm{c}}$, Victor A. Molina ${ }^{\mathrm{d}}$, \\ Cecilia Forcato $^{\mathrm{a}, 1}$, María E. Pedreira ${ }^{\mathrm{a}, *, 1}$

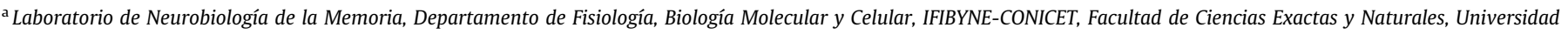 \\ de Buenos Aires, Argentina \\ ${ }^{\mathrm{b}}$ Departamento de Física, Facultad de Ciencias Exactas y Naturales, Universidad de Buenos Aires, Argentina \\ ${ }^{\mathrm{C}}$ Fundación para la Lucha contra las Enfermedades Neurológicas de la Infancia (FLENI), Buenos Aires, Argentina \\ ${ }^{\mathrm{d}}$ Departamento de Farmacología, IFEC/CONICET-UNC, Facultad de Ciencias Químicas, Córdoba, Argentina
}

\section{A R T I C L E I N F O}

\section{Article history:}

Received 1 April 2015

Revised 9 October 2015

Accepted 21 October 2015

Available online 7 November 2015

\section{Keywords:}

Neutral declarative memory

Memory improvement

Memory persistence

Pre-learning stress

Psychosocial stress

Autonomic nervous system

\begin{abstract}
A B S T R A C T
Memories can be altered by negative or arousing experiences due to the activation of the stressresponsive sympatho-adrenal-medullary axis (SYM). Here, we used a neutral declarative memory that was acquired during multi-trial training to determine the effect of a threatening event on memory without emotional valence. To this end, participants received a new threatening social protocol before learning pairs of meaningless syllables and were tested either $15 \mathrm{~min}, 2$ days or 8 days after acquisition. We first demonstrated that this threatening social situation activates not only the SYM axis (Experiment 1) and the hypothalamus-pituitary-adrenal axis (HPA; Experiment 2), but also, it improves the acquisition or early consolidation of the syllable pairs (Experiment 3 ). This improvement is not a transient effect; it can be observed after the memory is consolidated. Furthermore, this modulation increases the persistence of memory (Experiment 4). Thus, it is possible to affect memories with specific events that contain unrelated content and a different valence.
\end{abstract}

(c) 2015 Elsevier Inc. All rights reserved.

\section{Introduction}

Negative or arousing experiences can alter cognitive processes, such as memory (Diamond, Campbell, Park, Halonen, \& Zoladz, 2007). Exposure to a situation that is perceived as threatening or excessively demanding (i.e., a stressor) induces the release of hormones and other cellular mediators, which can promote adaptation and the onset of coping responses, thereby orchestrating an emotional response to the stressor (Joëls \& Baram, 2009). The effect on memory processes is in part due to the activation of the stressresponsive sympatho-adrenal-medullary (SYM) and hypothalamus-pituitary-adrenal (HPA) axes (Allen, Kennedy, Cryan, Dinan, \& Clarke, 2014).

Although negative emotional states may be elicited by a variety of circumstances, those that threaten a social goal and affect the

\footnotetext{
* Corresponding author at: Laboratorio de Neurobiología de la Memoria, Departamento de Fisiología, Biología Molecular y Celular, Facultad de Ciencias Exactas y Naturales, Universidad de Buenos Aires IFIByNE, CONICET. Ciudad Universitaria, Pab. II, 2do piso (C1428EHA), Buenos Aires, Argentina. Fax: +54 1145763447.

E-mail address: mpedreira@fbmc.fcen.uba.ar (M.E. Pedreira).

1 These authors contributed equally to this work
}

ability to overcome that threat produce a stress reaction (Lazarus, 1966). A social-evaluative threat occurs when an important aspect of self-identity is or could be negatively judged by others and the outcome of the situation is uncontrollable (Dickerson \& Kemeny, 2004). In the laboratory, the Trier Social Stress Test (TSST) is a protocol that can be particularly useful for inducing this type of response in humans (Kirschbaum, Pirke, \& Hellhammer, 1993).

It is commonly accepted that stress facilitates memories with emotional content but that it either impairs or has no effect on neutral information (Nater et al., 2007; Smeets, Giesbrecht, Jelicic, \& Merckelbach, 2007; Smeets, Otgaar, Candel, \& Wolf, 2008; Wolf, 2009); however, other reports have shown that stress may in fact improve neutral memory (Schwabe, Bohringer, Chatterjee, \& Schachinger, 2008).

We designed a multi-trial memory task that consisted of learning a list of five pairs of meaningless syllables (each pair was formed by a cue syllable that was associated with a response syllable (Forcato et al., 2007). In contrast with our paradigm, other tasks have employed a single training trial using an episodic or semantic memory with mixed content (emotional vs. neutral), which, in turn, is modified by the stressful situation (Dongaonkar, 
Hupbach, Gomez, \& Nadel, 2013). Here, the clear advantage was the use of a pure neutral declarative memory that was acquired during multi-trial training. During this training, it is possible to determine the inclusion criteria for the subjects' level of learning. Then, under this experimental condition, it was possible to analyze the effect of a threatening event on memory without emotional valence.

Based on the TSST, we designed a new threatening social protocol. In this original version, subjects interacted with a virtual auditory panel (VAP). This new protocol included a VAP that was generated by only one researcher, thereby reducing the costs and optimizing the organization of the experiment schedule. We first evaluated whether the VAP induced the activation of the SYM axis (Experiment 1) by administering the protocol following the acquisition of a neutral declarative memory. Then, we compared the effects of the VAP with those of the TSST protocol using the same physiological measures plus cortisol, thereby demonstrating a similar activation not only of the SYM axis but also of the HPA axis (Experiment 2). We next analyzed the VAP's effects on memory, by testing $15 \mathrm{~min}$ (short-term, Experiment 3), 2 days or 8 days after acquisition (long-term and forgetting, Experiment 4).

In doing so, we found that a threatening social situation that activates the SYM and HPA axes can modulate the acquisition and/or early consolidation of a non-related neutral declarative task. This modulation is not a transient effect; its consequences were observed after the memory was consolidated, and it modified the persistence of memory. Another important contribution is the possibility of studying the effects of a stressor on a pure neutral memory (with a controlled level of learning). This allows for a new line of studies in which researchers can develop their understanding of the relationship between this memory type and the physiological changes that are induced by stress.

\section{Methods}

One hundred and seventy-six undergraduate and graduate students from Buenos Aires University (Argentina) participated in the current study. Prior to the experiments, participants provided a written informed consent that was approved by the Ethics Committee of the Review Board of the National Society of Clinical Research (Sociedad Argentina de Investigación Clínica). The following students were excluded from the experiments: students with cardiovascular and endocrine diseases; students having physical illnesses or being on any kind of medication; female students with no normal menstrual cycles (28-30 days), taking contraceptive pills, or within the 1st-14th day of the menstrual cycle. Current or lifetime psychopathology and/or substance abuse were assessed by a clinical psychologist.

\subsection{Virtual-Auditory Panel (VAP) protocol}

We designed a threatening protocol to evaluate a negative emotional response in the participants before they learned a neutral memory task. The VAP protocol consisted of three phases (Fig. 1). Phase 1 was an undemanding attentional task, in which 16 landscape images were shown on the screen for $5 \mathrm{~s}$ each. The images appeared randomly and were presented in two blocks. Participants were asked to rate the images in a scale using the keyboard (from 1 to 5) according to their likes. This undemanding task lasted 5 min and was used as the skin conductance baseline level. In Phase 2, participants had to prepare a speech to advertise themselves as the best candidate for a professional position; this phase lasted 5 min. Finally, in Phase 3, the experimenter explained to the participants that a hospital committee was following the presentation online. Participants had to talk to a webcam which was located on the back wall; participants were provided with headphones and a microphone to deliver their speech. As in the TSST protocol (Kirschbaum et al., 1993), after the presentation, participants had to perform an arithmetic task. The experimenter used a pitch modifier provided with three different voices (virtual panel) that simulate a hospital committee. This virtual panel allowed a reliable environment using pre-recorded ambient sound (consisting of different office sounds such as engines, papers, keys, and chairs). Thus, the experimenter could interact with the participants in a way similar to the TSST.

Besides, we designed a non-threatening protocol, similar to the VAP but without the main stress components (such as social threat and uncontrollability) (Dickerson \& Kemeny, 2004). We called it fake VAP (VAPf). The first two phases of this protocol were identical to the previous protocol. In contrast, in Phase 3, participants had to write down the speech and resolve the arithmetic task. In order for both protocols to last the same time, we included other tasks such as different multiplications, additions or symbol translations.

The virtual panel software and the pre-recorded ambient sound were programmed in Cycling'74. Max/msp 5.0. All the sound devices (microphones and headphones) were plugged in a 5-channel stereo mixer (SKP Pro audio VZ 5 series) connected to the experimenter's computer, located in the contiguous room. All the wires were hidden from the subjects' view.

\subsubsection{Measurements}

Before Phase 1, we obtained the baseline measurements for the State Trait Anxiety Inventory (STAI), blood pressure, and heart rate. Blood pressure and heart rate were taken at four different time points: t0 (before Phase 1), t1 (after Phase 2), t2 (after the speech presentation) and t3 (after the arithmetic task) (Fig. 1). Skin conductance level (SCL) was recorded during the entire experiment; we defined the SCL baseline level as the continuous measure during Phase 1 (Fig. 1). At the end of Phase 3, blood pressure, heart rate and the STAI were measured for the last time. The STAI was measured 10 min after the end of the arithmetic task.

2.1.1.1. Subjective rating. Cognitive stress and anxiety were measured using the STAI (Spielberger, Gorsuch, \& Lushene, 1970) before and after the administration of the procedures (before Phase 1 and 10 min after Phase 3 respectively).

2.1.1.2. Blood pressure (Systolic Blood Pressure (SBP) and Diastolic Blood Pressure (DBP)) and Heart Rate (HR). Blood pressures were assessed using an Omron HEM 7220 Premium digital Tensiometer (http://omronhealthcare.com/products/7-series-upper-arm-bloodpressure-monitor-bp760/). Cardiovascular measurements were taken before Phase $1(\mathrm{t} 0)$, after Phase $1(\mathrm{t} 1)$, at the end of the speech (t2), and at the end of the arithmetical task (t3) (Fig. 1)

2.1.1.3. Electrodermal activity. Electrodermal activity was measured along the entire experiment, using an input device with a sineshaped excitation voltage $( \pm 0.5 \mathrm{~V})$ of $50 \mathrm{~Hz}$, derived from the main frequency used in Psychlab Precision Contact Instruments (http:// www.psychlab.com/). The input device was connected to two Ag/ $\mathrm{AgCl}$ electrodes of $20 \mathrm{~mm} \times 16 \mathrm{~mm}$. The electrodes were located in the intermediate phalanges of the index and middle fingers of the non-dominant hand. Data were analyzed with Matlab (Mathworks Inc. Sherborn, MA, USA) and Ledalab (Benedek \& Kaernbach, 2010).

2.1.1.4. Neuroendocrine response to HPA axis activity. Free salivary cortisol was measured in all groups before Phase 1 and $10 \mathrm{~min}$ after Phase 3. Samples were collected using Salivette sampling 


\author{
I Baseline Measures \\ STAI-S \\ Salivary Cortisol \\ Blood Preassure + Heart Rate (to) \\ Electrodermal Activity
}

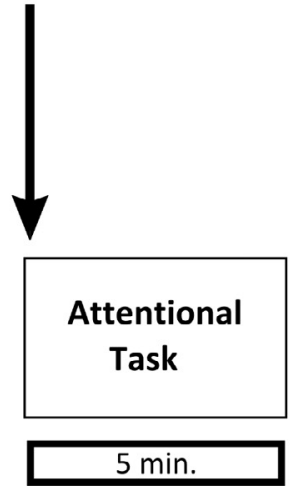

Phase I

\section{Measures}

Blood Preassure + Heart Rate (t1)
III Measures

Blood Preassure + Heart Rate (t2)

Electrodermal Activity

\section{Measures}

Blood Preassure + Heart Rate (t3)

Electrodermal Activity

STAI-S (10 min. after)

Salivary Cortisol (10 min. after)

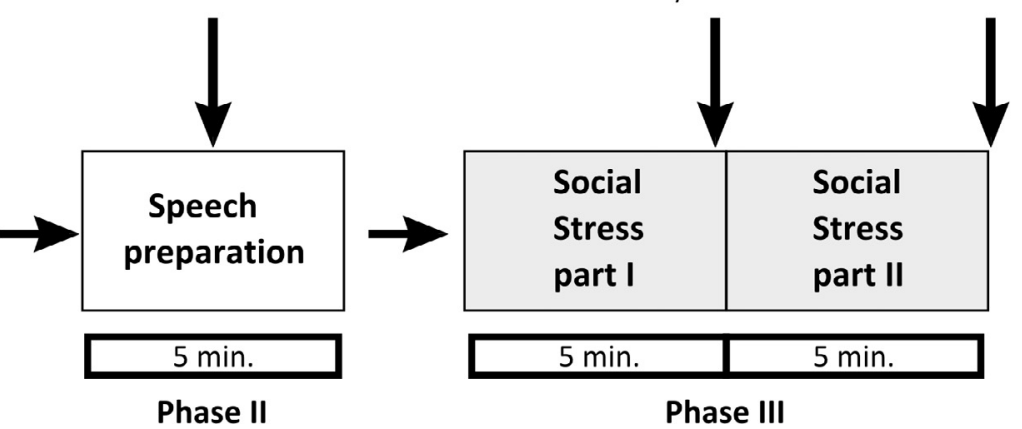

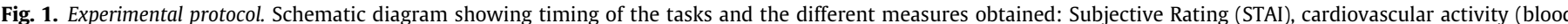

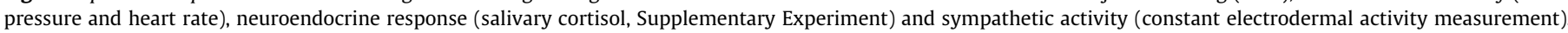

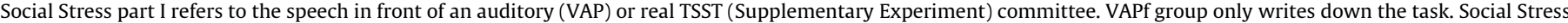
part II stands for the arithmetic task and was conducted as above. The arrows stand for the time when the measures were taken.

devices and they were stored at $-20^{\circ} \mathrm{C}$. Salivary cortisol levels were determined with luminescence immunoassay in FLENI.

\subsection{Memory protocol}

We studied the effect of the VAP and VAPf on a neutral declarative memory. We used five pairs of meaningless syllables presented in an enriched specific context (image, colored light and music) as reported in previous work from our laboratory (Forcato, Fernandez, \& Pedreira, 2013; Forcato et al., 2007). Each pair was formed by a cue syllable associated with a response syllable (Fig. 2). During the training session, the list was presented in 10 different trials (in the first trial, participants saw the pairs and in the other nine trials they completed the pairs using the keyboard and received feedback). The testing session took place $15 \mathrm{~min}$ (short-term), 2 days (long-term) or 8 days (forgetting) after training and consisted of one presentation of the cue syllables without any kind of feedback.

Errors made at testing were classified as: Void type errors (blank responses), Confusion type errors (writing a non-existent response syllable) and the Intralist type (writing response syllables for a different cue syllable).

Additionally to the exclusion criteria mentioned above, only the subjects that achieved at least $65 \%$ of correct responses during the last four trails of the training session (13/20 correct responses) were included.

\subsection{Experiment 1}

\subsubsection{Participants}

Ninety-eight healthy subjects participated in Experiment 1 ( $24 \pm 2.5$ years old, 60 females and 38 males). The VAP group ( $n=49,24 \pm 2.1$ years old) included 31 females and 18 males, whereas the VAPf group ( $n=49,24 \pm 2.3$ years old) included 29 females and 20 males. The experiment was conducted between 9:00 a.m. and 3:00 p.m. The participants were split in both groups to balance the time of the day of the experiment.

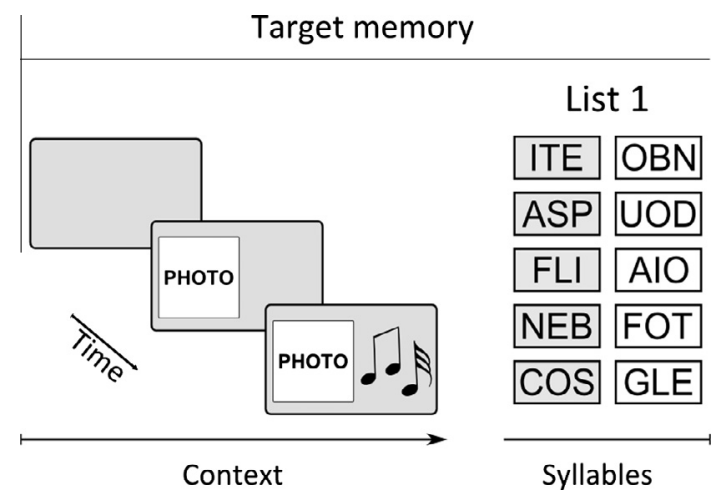

Fig. 2. Memory task. A trial consisted of the context period, i.e. a specific combination of a light (color illumination of the room), image (a picture) and sound (music), and by a syllable period, i.e. six seconds after the stimulus presentation, the five pairs of cue-response syllables (List 1 as shown) were presented successively 10 times in random order. Testing session consisted in the context formation and only one cue recall trial.

\subsubsection{Experimental protocols}

We compared the physiological measurements obtained with the VAP and VAPf protocols. We contrasted cardiovascular data, subjective ratings and sympathetic activity.

\subsection{Experiment 2}

\subsubsection{Participants}

Forty-five subjects ( $25 \pm 2.8$ years old, 12 males and 18 females) volunteered for the study. The TSST included 7 males and 8 females ( $25 \pm 2.2$ years old), the VAP7 males and 8 females ( $25 \pm 2.5$ years old) and the VAPf6 males and 9 females ( $24 \pm 3.1$ years old).

\subsubsection{Experimental protocols}

The study used the same two experimental protocols as in Experiment 1 with the addition of the TSST. Under the TSST, Phase 1 and Phase 2 were the same as in VAP and VAPf procedures. In the Phase 3, participants were standing before a committee of two professionals from a hospital, (a psychologist and a psychiatrist) 
dressed in white coats. Their speech had to last $5 \mathrm{~min}$. The committee had to look indifferent or little interested in the presentation, they asked the participants to continue talking or they posed general questions to complete the time limit. Then, the participants were instructed to perform an arithmetic task. It consisted in subtracting 13 from 1022, and then sequentially subtracting 13 again from the result as fast and accurately as possible within a $5 \mathrm{~min}$ span. When failing or delaying an answer, they had to restart from 1022 (Kirschbaum et al., 1993).

\subsubsection{Measures}

The same measures from Experiment 1 were taken with the addition of salivary cortisol.

\subsection{Experiment 3}

\subsubsection{Participants}

Thirty-three participants ( $25 \pm 2.2$ years old; 18 females and 15 males) participated in Experiment 2. The VAP group included 6 females and 5 males ( $24 \pm 2.5$ years old), the VAPf group included 5 females and 6 males ( $25 \pm 2.1$ years old) and the control group included 7 females and 4 males ( $25 \pm 2.3$ years old). The experiment was conducted between 9:00 a.m. and 3:00 p.m. The participants were split in the three groups to balance the time of the day of the experiment.

\subsubsection{Experimental protocols and measurements}

We aimed to test the effect of the VAP and VAPf on the acquisition of a neutral declarative memory. This experiment included the threatening and non-threatening protocols plus a control group that performed only the memory task. The physiological measurements were equal to those of Experiment 1 . The training session started immediately after the last STAI measurement. The Testing session took place 15 min after training.

\subsection{Experiment 4}

\subsubsection{Participants}

Fifty participants ( $24 \pm 2$ years old; 29 females and 21 males) participated in Experiment 3. The VAP $3 \mathrm{~d}$ group included 8 females and 4 males, the Control 3d group included 7 females and 6 males, the VAP 8d group included 6 females and 7 males, and the Control $8 \mathrm{~d}$ group included 8 females and 4 males. The experiment was conducted as above.

\subsubsection{Experimental protocols and measurements}

We studied the effect of the VAP on a neutral declarative memory at long-term retention and forgetting. This experiment included the threatening protocol plus a control group that performed only the memory task. The physiological measurements were equal to those of Experiment 1. The Training session was as in Experiment 2. The Testing session took place 2 days after training (VAP 3d and Control 3d groups) and 8 days after for the VAP $8 d$ and Control 8d groups.

\subsection{Statistical analysis}

\subsubsection{STAI}

Is reported as the mean score difference in each participant at the end of (Phase 3 ) and before the attentional task (Phase 1). Data were analyzed using one-way analysis of variance (ANOVA), followed by LSD post-hoc comparisons (FISHER, $\alpha=0.05$ ) when necessary.

\subsubsection{Blood pressure and heart rate}

A mean cardiovascular value $(\mathrm{t} 0, \mathrm{t} 1, \mathrm{t} 2, \mathrm{t} 3)$ was reported $(\mathrm{mm} /$ HG, BPM). Data were analyzed using repeated measures ANOVA (Group $\times$ Time). When the interaction was significant, simple effects were performed followed by LSD comparisons when appropriate. When sphericity was not accomplished, GreenhouseGeisser correction was applied.

\subsubsection{Electrodermal activity}

SCL is reported as the mean SCL $(\mu S)$ in each participant during the baseline attentional task (Phase 1 ) and during stress induction (Phase 3). The use of the mean SCL is supported by the stationary time series of the signal and the low variability between points (Supplementary Fig. 1). Data were analyzed using repeated measures ANOVA (Group $\times$ Time). Interaction, post-hoc comparisons and sphericity were treated as with blood pressure measurements and heart rate.

\subsubsection{Neuroendocrine response to HPA axis activity}

It was reported as the mean cortisol level ( $\mathrm{nmol} / \mathrm{l})$ for each participant before the attentional task (Phase 1 ) and at the end of the test (Phase 3). Data was analyzed as cardiovascular activity.

\subsubsection{Neutral declarative memory}

The Training session is reported as the mean number of errors per training trial and was analyzed with repeated measures ANOVA. The Testing session was first analyzed with one-way ANOVA and followed by post-hoc comparisons (FISHER, $\alpha=0.05$ ). When significant results were obtained, Partial Eta Squared $\left(\eta_{p}{ }^{2}\right)$ was used as an effect size measure in all the experiments. Typically, a $\eta_{p}{ }^{2}=0.01$ represents a small effect, a $\eta_{p}{ }^{2}=0.06$ represents a medium effect and a $\eta_{p}{ }^{2}=0.14$ represents a large effect (Fritz, Morris, \& Richler, 2012). We also studied the different types of errors (Forcato et al., 2013). Void, Intralist and Confusion-Types errors are reported as the mean number of errors and were analyzed with one-way ANOVA, followed by LSD post-hoc comparisons (FISHER, $\alpha=0.05$ ).

\section{Results}

\subsection{Experiment 1}

The aim of experiment 1 was to develop and evaluate the effects of the VAP protocol by using the activation of the SYM axis and reported stress as measures of efficacy against a control condition that did not include the stressful components (Dickerson \& Kemeny, 2004).

\subsubsection{Cardiovascular response}

The VAP group showed higher SBP than that of the VAPf group at $\mathrm{t} 2$ and $\mathrm{t} 3$. We found no differences between groups at $\mathrm{t} 0$ or $\mathrm{t} 1$ (Fig. 3A.1, repeated measures ANOVA, Group $\times$ Time interaction $F$ $\left.(3,28)=54.20, p<0.001, \eta_{p}{ }^{2}=0.37\right)$. The SBP increased for the VAP group between $\mathrm{t} 1$ and $\mathrm{t} 2$ and then remained unchanged until the end of the procedure $(t 3)(p<0.001)$. We found no differences within the VAPf over time ( $\mathrm{t} 0, \mathrm{t} 1, \mathrm{t} 2, \mathrm{t} 3)$. In conclusion, we found no differences between or within groups at Phase 1 or Phase $2(\mathrm{t} 0, \mathrm{t} 1)$, thereby indicating the specificity of stress induction at Phase $3(\mathrm{t} 2$ and $\mathrm{t} 3$ ). Similar results were found for DBP (repeated measures ANOVA, Group $\times$ Time interaction $F(3,28)=61.05, p<0.001$, $\eta_{p}{ }^{2}=0.39$ ) and HR (repeated measures ANOVA, Group $\times$ Time interaction $F(3,28)=25.04, p<0.001, \eta_{p}{ }^{2}=0.20$, Fig. $3 A .2$ and A.3). The VAP group showed a higher DBP and HR than did the VAPf group during the stress procedure $(t 2, t 3)$. Here again, DBP and HR increased significantly in the VAP group between $\mathrm{t} 1$ and $\mathrm{t} 2$. 
A Cardiovascular Data

\section{A.1 Systolic Blood Pressure}

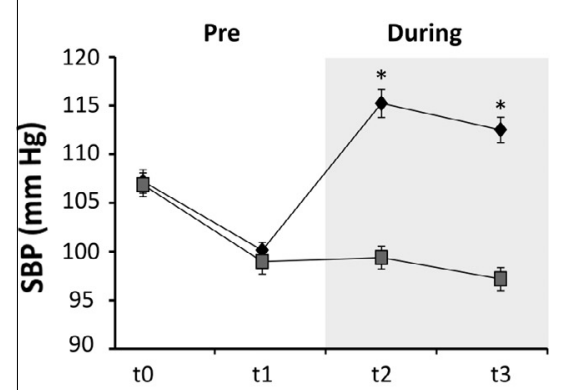

A.2 Diastolic Blood Pressure

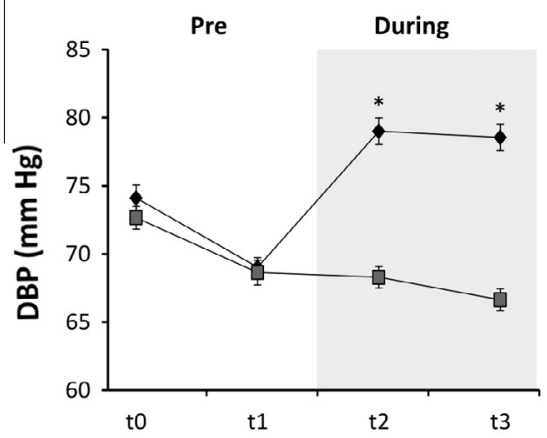

A.3 Heart Rate

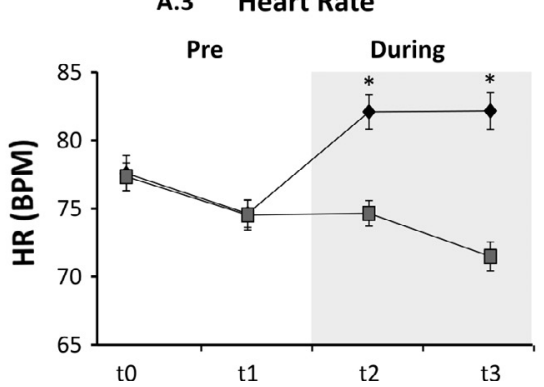

B Subjetive Rating

Mean STAI-S Difference

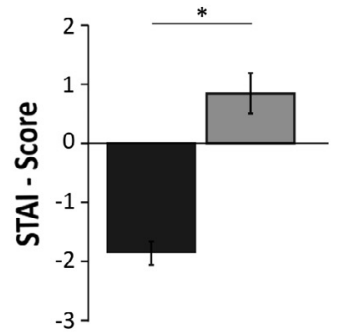

C Sympathetic Activity

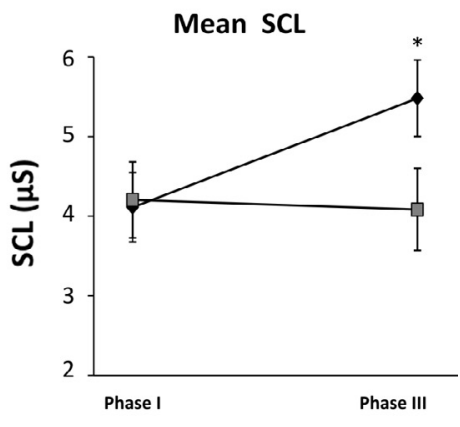

VAP

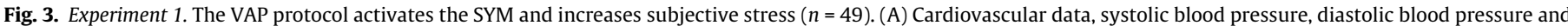

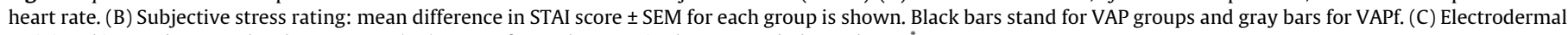
activity skin conductance level: Mean SCL $(\mu S) \pm$ SEM for each group is shown. Symbols as above. ${ }^{*}, p<0.05$.

\subsubsection{Subjective rating}

The VAP group showed a significantly lower STAI score than did the VAPf, which indicates an increase in subjective stress (Fig. 3B, one-way ANOVA $F(1,96)=45.29, p<0.001, \eta_{p}^{2}=0.32$ ).

\subsubsection{Sympathetic activity}

SCL was higher during Phase 3 in the VAP group than in the VAPf group (Fig. 3C, repeated measures ANOVA Group $\times$ Time interaction, $F(1,96)=85.03, p<0.001, \eta_{p}{ }^{2}=0.49$, simple effects $p<0.001$ ). The VAP group also showed a significant increase in SCL between Phase 1 and Phase 3 (simple effects $p<0.001$ ). In summary, these results suggest that the VAP protocol induced a significant increase in subjective stress and the SYM response, which was maintained until the end of the procedure, as indicated by variations in the cardiovascular response and electrodermal activity.

\subsection{Experiment 2}

As previously discussed, Experiment 1 was used to demonstrate that the VAP protocol can induce a possible stress response, as indicated by strong SYM activation and subjective stress. Given that the other major component of the threat response is the activation of the HPA axis and that the TSST is known to reflect not only SYM activation but also the HPA axis variations, we compared this protocol with the VAP and VAPf. Thus, we repeated the same SYM and subjective measurements as those in Experiment 1 with the addition of salivary cortisol as an index of the HPA axis.

\subsubsection{Cardiovascular response}

The TSST and VAP groups showed higher SBP than the VAPf group at $\mathrm{t} 2$ and $\mathrm{t} 3$ (Table 1 , middle panel a; repeated measures ANOVA, Group $\times$ Time interaction $F(6,81)=6.77 p<0.001$, $\left.\eta_{p}{ }^{2}=0.33\right)$. No difference between groups was observed at t0 or at 11 (simple effects $p=0.966, p=0.784$, respectively). SBP was increased for the groups TSST and VAP but not for the VAPf, between $\mathrm{t} 1$ and $\mathrm{t} 2$. This observation persisted until the end of the procedure ( $t 3$; Fig. $4 \mathrm{~A}$ ).

Similar results were found for DBL (Table 1 , middle panel $b$; repeated measures ANOVA, Group $\times$ Time interaction $F(6,81)=$ 6.05, $p<0.001, \eta_{p}{ }^{2}=0.30$ ). The TSST and VAP Groups showed a 
Table 1

Cognitive and physiological measures for the Experiment 1 . Cardiovascular activity at different time points (t0, t1, t2, t3). Mean SBP (mmHg), mean DBP $(\mathrm{mmHg})$ and mean HR (BPM), mean subjective rating difference and SCL $(\mu \mathrm{S})$ ( \pm SEM at 4 different time points for the three groups. The $F$ and effect size reported corresponds to the Group $\times$ Time interaction of a repeated measures ANOVA. SE stands for simple effects.

\begin{tabular}{|c|c|c|c|}
\hline & \multicolumn{3}{|c|}{ Means ( \pm SE) of the cognitive and physiological measures } \\
\hline & \multicolumn{2}{|l|}{ Experiment 1} & \\
\hline & VAPf & VAP & \\
\hline \multicolumn{4}{|c|}{ Systolic BP ( $\mathrm{mmHg})$} \\
\hline to & $106.85(1.22)$ & $107.20(1.21)$ & $F(3,28)=54.20$ \\
\hline $\mathrm{t} 1$ & $98.97(1.28)$ & $100.12(0.82)$ & $p<0.001 ; \eta_{p}^{2}=0.37$ \\
\hline $\mathrm{t} 2$ & 99.38 (1.17) & $115.22(1.44)$ & SE $p_{\mathrm{t} 2}<0.001 ; \mathrm{SE} p_{\mathrm{t} 3}<0.001$ \\
\hline t3 & $97.18(1.18)$ & $112.48(1.31)$ & SE VAP $p_{\mathrm{t} 1-\mathrm{t} 2}<0.001$ \\
\hline \multicolumn{4}{|c|}{ Diastolic BP (mmHg) } \\
\hline to & $72.65(0.84)$ & $74.10(0.96)$ & $F(3.28)=61.05$ \\
\hline $\mathrm{t} 1$ & $8.63(0.91)$ & $69.02(0.71)$ & $p<0.001 ; \eta_{p}^{2}=0.39$ \\
\hline $\mathrm{t} 2$ & $68.28(0.79)$ & $79(0.97)$ & $\mathrm{SE} p_{\mathrm{t} 2}<0.001 ; \mathrm{SE} p_{\mathrm{t} 3}<0.001$ \\
\hline 3 & $66.63(0.80)$ & $78.55(0.96)$ & SE VAP $p_{\mathrm{t} 2-\mathrm{t} 3}<0.001$ \\
\hline \multicolumn{4}{|l|}{$H R(B P M)$} \\
\hline to & $77.34(1.02)$ & $77.59(1.32)$ & $F(3.28)=25.04$ \\
\hline $\mathrm{t} 1$ & $74.53(1.09)$ & $74.63(1.01)$ & $p<0.001 ; \eta_{p}^{2}=0.20$ \\
\hline $\mathrm{t} 2$ & $74.65(0.93)$ & $82.10(1.26)$ & $\mathrm{SE} p_{\mathrm{t} 2}<0.001 ; \mathrm{SE} p_{\mathrm{t} 3}<0.001$ \\
\hline t3 & $71.48(1.07)$ & $82.16(1.33)$ & SE VAP $p_{\mathrm{t} 2-13}<0.001$ \\
\hline \multicolumn{4}{|l|}{ Subjective rating } \\
\hline STAI difference & $0.67(0.19)$ & $-1.63(0.28)$ & $F(1.96)=45.29 p<0.001, \eta_{p}^{2}=0.32$ \\
\hline \multicolumn{4}{|l|}{$S C L(\mu S)$} \\
\hline Phase I & $3.96(0.22)$ & $3.90(0.21)$ & $F(1,96)=85.03, p<0.001$ \\
\hline Phase III & $4.00(0.24)$ & $5.53(0.22)$ & $\eta_{p}^{2}=0.49 ;$ SE $p<0.001$ \\
\hline
\end{tabular}

Values printed in bold denote significant within or between group differences.

significant increment in DBL than did the VAPf Group during the stress procedure $(t 2, t 3)$. As with SBP, the DBP for the TSST and VAP groups significantly increased between $t 1$ and $t 2$.

Finally, the TSST and VAP groups showed an increment in HR between $\mathrm{t} 1$ and $\mathrm{t} 2$ (Table 1 , middle panel $\mathrm{c}$; repeated measures ANOVA, interaction $\left.F(6,81)=3.24, p<0.02, \eta_{p}{ }^{2}=0.19\right)$. Additionally, the HR of the different groups showed no differences at t0, $\mathrm{t} 1$ and $\mathrm{t} 2$ (Table 1, middle panel c). While the VAP group showed a higher HR than did the VAPf at $\mathrm{t} 3$, no differences between the VAP and TSST groups were found.

Thus, the VAP induced SBP, DBP and HR increases that were similar to those obtained for the TSST.

\subsubsection{Subjective rating}

Fig. 4B shows STAI score differences between pre- and poststress induction for the three groups. A one-way ANOVA indicated a significant reduction in the score that was obtained for TSST and VAP but not for the VAPf group $(F(2,42)=8.418, p<0.001$, $\eta_{p}{ }^{2}=0.28$; LSD post-hoc Comparison $p=0.005, p=0.04$, respectively). There were no significant differences between the TSST and VAP, which showed similar effects between both protocols $(p=0.68)$.

\subsubsection{Neuroendocrine response}

The salivary cortisol levels between the baseline and the final time point of the VAP, TSST and VAPf can be observed in Fig. 4C. Salivary cortisol was higher under the TSST and VAP when compared to the VAPf group during Phase 3 (repeated measures ANOVA, Group $\times$ Time interaction $F(2,42)=7.87, p<0.001$, $\eta_{p}{ }^{2}=0.27$; simple effects, $p<0.001$, post-hoc LSD $p_{\text {VAP-VAPf }}=0.007$, $p_{\text {TST-VAPf }}=0.009, p_{\text {VAP-TSST }}=0.90$ ). There was a significant increase in cortisol levels between Phase 1 and Phase 3 for the VAP and TSST groups (simple effects VAP $p<0.001$; LSD $p_{\mathrm{VAP}}<0.001$; simple effects TSST $p<0.001$, LSD $p_{\text {TSST }}<0.001$ ). Therefore, the VAP produced an HPA axis response that was similar to that produced by the TSST.

\subsubsection{Sympathetic activity}

SCL analysis showed an increase in the signal in the stress groups (Fig. 4D, TSST and VAP) compared to the VAPf during Phase 3 (Repeated measures ANOVA, Group $\times$ Time interaction $F(2,42)=$ 9.89, $p<0.001, \eta_{p}{ }^{2}=0.32$; simple effects $p<0.001$, post-hoc LSD, $\left.p_{\text {VAP-VAPf }}<0.005, p_{\text {TSST-VAPf }}<0.05, p_{\text {VAP-TSST }}=0.59\right)$ and between Phase 1 and Phase 3 (simple effects VAP $p<0.001$; LSD $p_{\mathrm{VAP}}<0.001$; simple effects TSST $p<0.001$, LSD $p_{\text {TSST }}<0.001$ ). Here, again, the TSST and the VAP showed a comparable response of SYM activation.

\subsubsection{Analysis of different biomarker correlations}

The goal of the following analysis was to investigate the correlation between the measures of the three different protocols. To perform the analysis, we considered the differences between Phase 3 and Phase 1 for each biomarker. In each case, the correlation was assessed using Pearson's test. This test showed a non-significant correlation between cortisol and HR, SBP, DBP or STAI (Fig. 4E.1). Conversely, a significant positive correlation between skin conductance (SCL) and cortisol was shown ( $r=0.48, p=0.01$, Fig. 4E.2). Therefore, only the SCL showed a correlation with cortisol variations, which suggests that SCL is an adequate biomarker for monitoring the stressful effect of VAP.

Based on these results, we decided to evaluate the effect of VAP through the subjective stress and SYM activation measures because the SCL biomarker might be a predictor of HPA axis variations. Then, having demonstrated in Experiments 1 and 2 that the VAP induced a significant threat response similar to that obtained by the TSST, we designed three different experiments to evaluate the effects of the VAP protocol that is administered before the acquisition of a neutral declarative memory. We evaluated the memory task at three different time points: 15 min (Experiment 3 ), 2 days and 8 days after training (Experiment 4 ).

\subsection{Experiment 3}

Subjects were trained with a list of neutral pairs of meaningless syllables (List 1, Fig. 2) and tested 15 min later. Groups differed in 
A

Cardiovascular Data

A.1

\section{Systolic Blood Pressure}

Pre

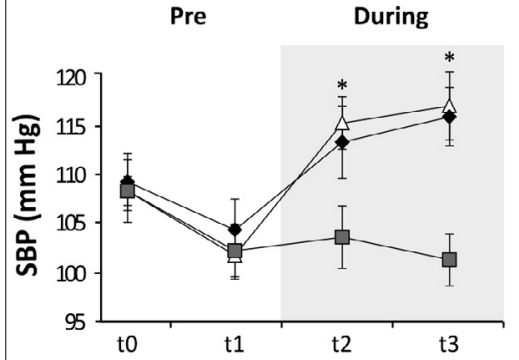

B Subjetive Rating Mean STAI-S Difference

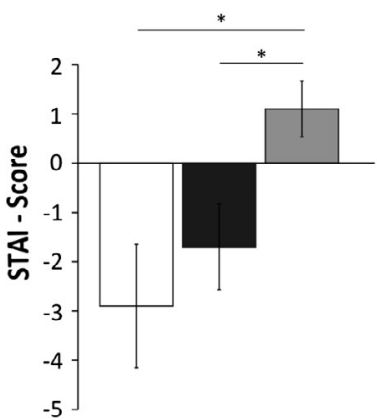

D Sympathetic Activity

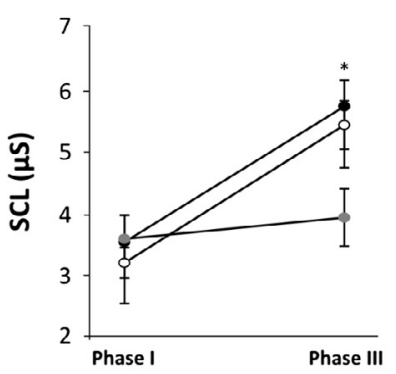

C Neuroendocrine Response

Mean Salivary Cortisol

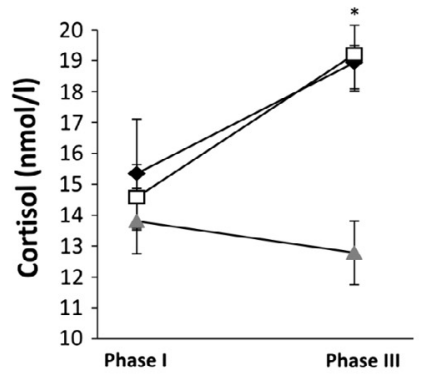

A.2 Diastolic Blood Pressure

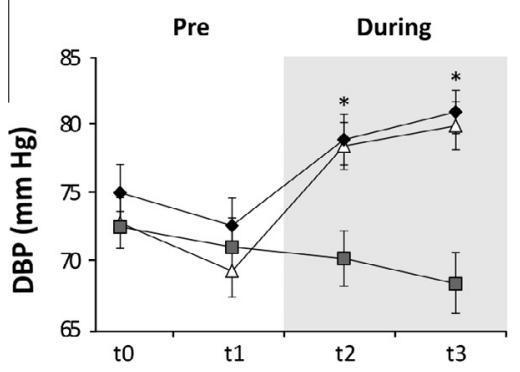

A.3 Heart Rate

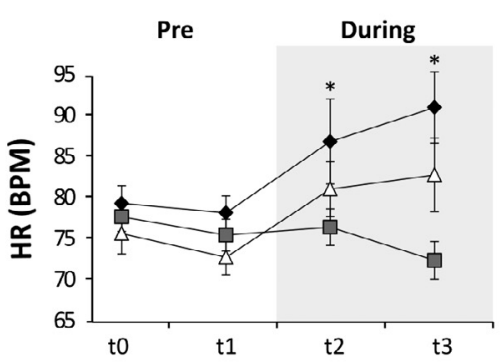

E.2

E.1

\begin{tabular}{|c|c|c|c|c|c|}
\hline & $\triangle \mathrm{SBP}(\mathrm{mm} \mathrm{Hg})$ & $\triangle \mathrm{DBP}(\mathrm{mm} \mathrm{Hg})$ & $\Delta \mathrm{HR}(\mathrm{BPM})$ & $\triangle$ STAI-S & $\Delta \mathrm{SCL}(\mu \mathrm{S})$ \\
\hline \multirow{2}{*}{$\begin{array}{c}\Delta \text { Cortisol } \\
(\mathrm{nmol} / \mathrm{l})\end{array}$} & $r=0,26$ & $r=0,23$ & $r=0,27$ & $r=-0,06$ & $r=0,48$ \\
\hline & $p=0,09$ & $p=0,11$ & $p=0,70$ & $\mathrm{p}=0,68$ & $p=0,01$ \\
\hline
\end{tabular}

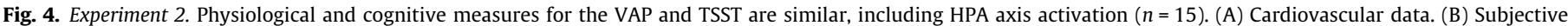

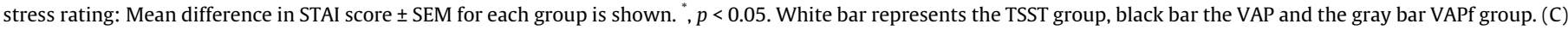

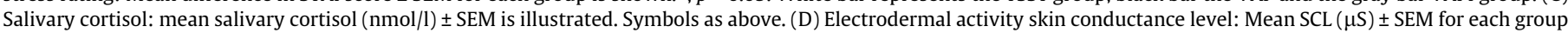

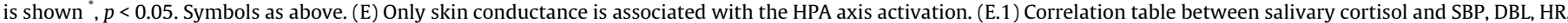

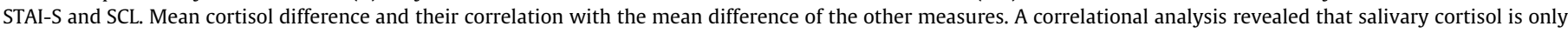

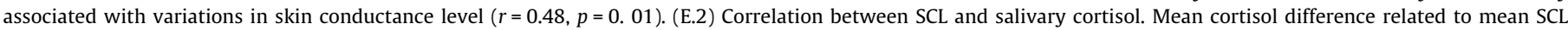
difference for each subject is shown. Pearson correlation coefficient $(r)$ and $p$ value of the regression analysis are shown.

the treatment they received before the training session. One group received the VAP, another group received the VAPf and the third group did not receive any treatment (VAP, VAPf and control groups, respectively, Fig. 5A.1).

\subsubsection{Cognitive and physiological measurements}

The VAP and VAPf groups showed the same profile as in Experiment 1 (Table 1 , middle panel).

\subsubsection{Memory task}

The VAP group made fewer errors than the VAPf and the control groups at the testing session (Fig. 5A.2, one-way ANOVA $F$ $(2,30)=3.57, p=0.03 ; \quad \eta_{p}{ }^{2}=0.20$ LSD post-hoc comparison $p=0.03$ and $p=0.02$, respectively). Considering the type of errors made during testing, the VAP group showed fewer void-type errors than did the control and VAPf groups (Fig. 5A.3, one-way
ANOVA $F(2,30)=3.35 p=0.04 ; \eta_{p}^{2}=0.19$, LSD post-hoc comparison $p=0.03$ and $p=0.03$, respectively); however, no differences between groups were found for confusion-type or intra-list-type errors (all $p>0.05$ ).

Finally, we analyzed the correlation between the percentage of memory change between acquisition and testing and the mean SCL difference during the VAP and VAPf protocols (Fig. 5B). We found a significant correlation between the change in memory performance and the SYM, as reflected by the SCL $(r=0.49 ; p=0.02)$. Moreover, the repeated-measures ANOVA revealed no significant differences between the groups during List 1 training (Fig. 5C, $F$ $(2,30)=0.65, p=0.59)$ or by trial interaction $(F(16,24)=0.27$, $p=0.99$ ). An analysis of the percentage of correct responses provided during the last four training trials yielded no significant difference between groups at training (Fig. 5C inset, $F(2,30)=$ $0.41, p=0.66$ ). These results show that a threatening social event 
A 1

\begin{tabular}{|c|c|c|}
\hline Groups & \multicolumn{2}{|c|}{ Day 1} \\
\hline VAP & VAP -- L1-TR & $\stackrel{15 \mathrm{~min}}{\longrightarrow} \mathrm{L} 1-\mathrm{TS}$ \\
\hline VAPf & VAPf -- L1-TR & $\stackrel{15 \mathrm{~min} .}{\longrightarrow} \mathrm{L} 1-\mathrm{TS}$ \\
\hline Ctl & L1-TR & $\stackrel{15 \mathrm{~min} .}{\longrightarrow}$ L1-TS \\
\hline
\end{tabular}

A.2

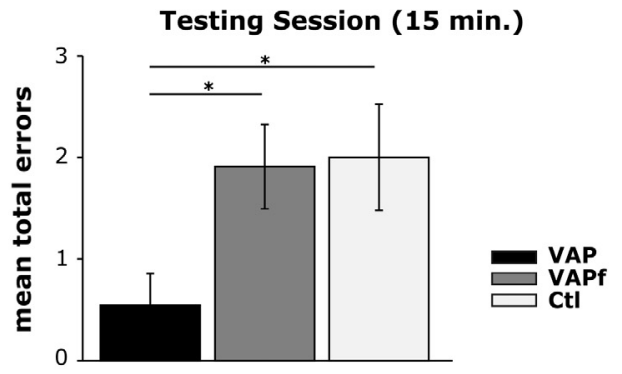

A.3

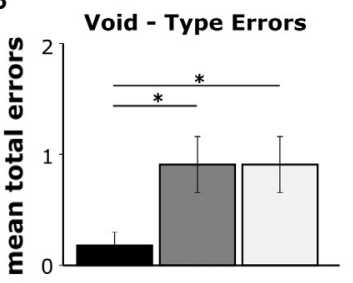

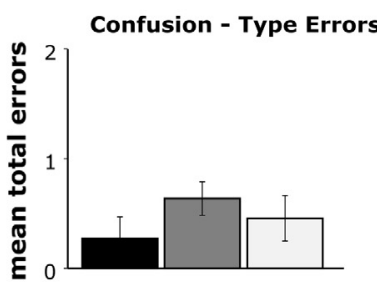

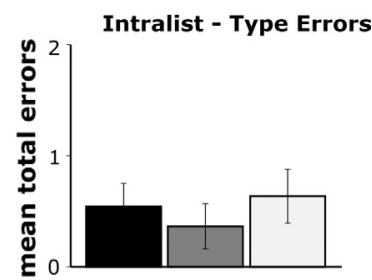

B

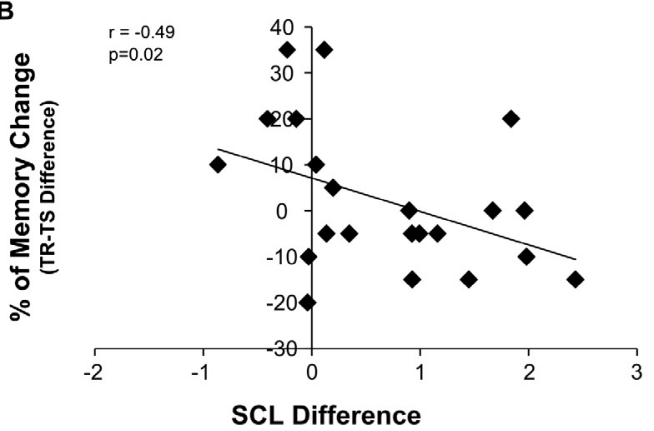

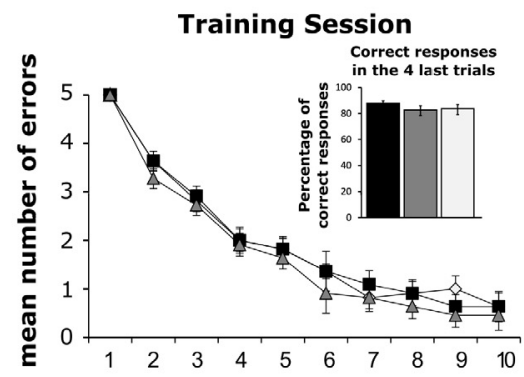

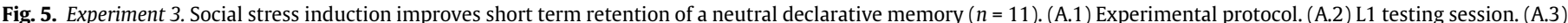

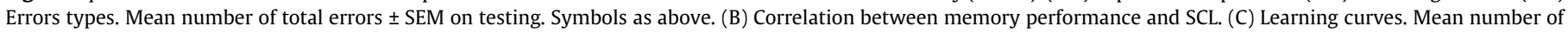
errors \pm SEM per trial on Day 1 black bar stands for VAP group, white bar for VAPf and gray bar for Ctl.

prior to learning improves the retention of a neutral declarative memory but has no effect during training.

\subsection{Experiment 4}

Subjects were trained on day 1 and tested either 3 or 8 days after acquisition. Prior to training, subjects either received the VAP treatment or no treatment (VAP 3d, Control 3d, VAP 8d and Control 8d, respectively, Fig. 6A).

\subsubsection{Cognitive and physiological measurements}

As expected, VAP 3d and VAP 8d repeated the same profile as in Experiments 1 and 2 (Table 1 ).

\subsubsection{Memory task}

As in the previous experiment, the VAP groups performed better than did the control groups (Fig. 6A.2, $F(2,30)=0.65$, $p<0.005, \eta_{p}{ }^{2}=0.36$, LSD post-hoc comparison $p_{\mathrm{VAP} 3 \mathrm{~d}-\mathrm{Ct} 3 \mathrm{~d}}<0.001$ and $p_{\mathrm{VAP}} 8 \mathrm{~d}-\mathrm{Ctl} 8 \mathrm{~d}=0.02$, respectively). With respect to void-type errors, the VAP $8 \mathrm{~d}$ group made fewer errors than did the Control 8d group (Fig. 6A.3, one-way ANOVA $F(3,46)=4.49, p=0.007$ $\eta_{p}{ }^{2}=0.31$; LSD post-hoc comparison $p=0.01$ ). There was no difference for the other types of errors (all $p>0.05$ ). In addition, the Control 3d had fewer errors than did the Control 8d, and this difference was also observed for the void-type errors (LSD posthoc comparison $p=0.04$ and $p=0.04$, respectively). This reflects the effect of forgetting. The VAP groups showed no significant correlation between the percentage of memory change and the mean SCL difference (VAP $3 d r=0.2 ; p=0.52$ and VAP $8 d$ $r=0.37, p=0.20)$. The repeated-measures ANOVA revealed no significant differences between the groups at List 1 training (Fig. $6 \mathrm{~B}, \quad F(3,45)=0.93, \quad p=0.43$ ) or by trial interaction $(F(24,360)=1.10, p=0.33)$. The percentage of correct responses that were provided during the last four training trials yielded no significant differences between groups at training (Fig. 6B inset $F(3,46)=5.64, p=0.66)$.

These results show that exposure to a threatening social event prior to learning not only improved the retention of a neutral declarative memory in the short- and long-term, but it also prevented forgetting. 
A

A.1

\begin{tabular}{cccc}
\hline Groups & Day 1 & Day 3 & Day 8 \\
\hline VAP 3d & VAP - L1-TR & L1-TS & --- \\
Ctl 3d & L1-TR & L1-TS & --- \\
VAP 8d & VAP -- L1-TR & --- & L1-TS \\
Ctl 8d & L1-TR & --- & L1-TS
\end{tabular}

A.2

Testing Session

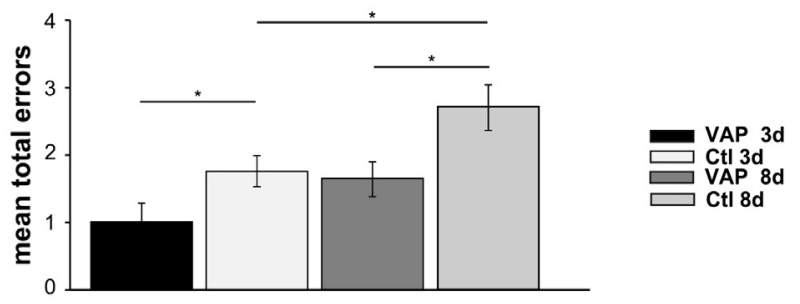

A.3
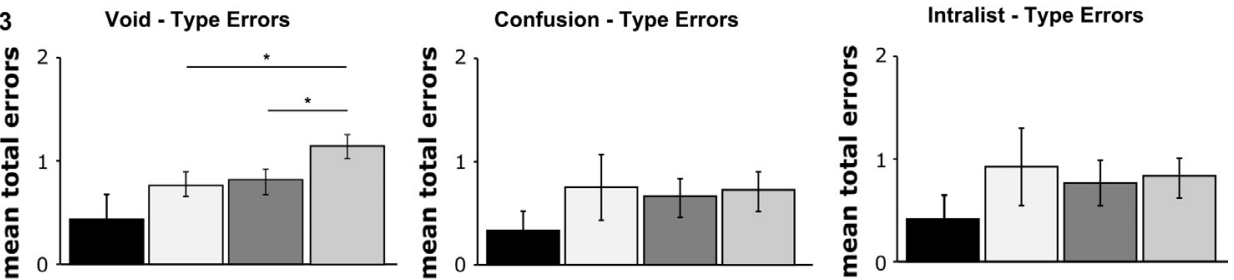

B

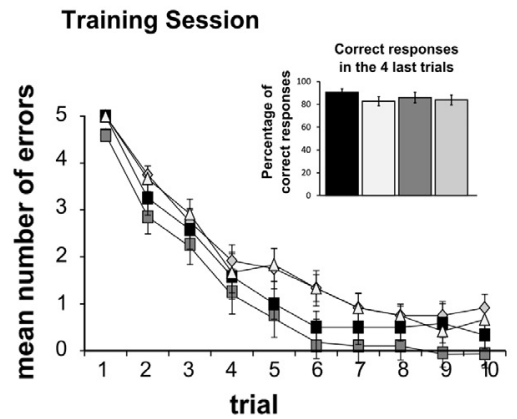

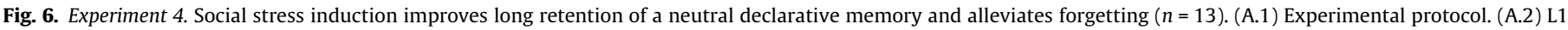

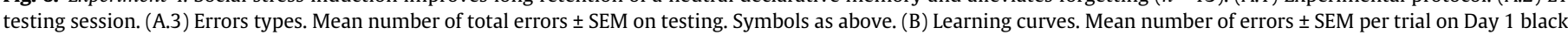
bar stands for VAP 3d group, white bar for Ctl 3d, dark gray for VAP 8d and gray bar for Ctl 8d.

\section{Discussion}

Here, we showed that a threatening social event that was not specifically related to the associative memory task can modulate the retention of a non-emotional declarative memory in both the short- and long-term and prevents forgetting. Thus, memories may be affected by specific events of unrelated content and different emotional valence. In addition, the performance on the shortterm test correlates with the arousal generated by the activation of the SYM axis. Although our experiments showed no differences between groups in the multi-trial training, we hypothesized that the memory changes observed 2 and 8 days after acquisition are a consequence of enhanced encoding. The memory task used in this report is quite different from those employed in other studies. In these studies, researchers evaluated memories for single neutral or emotional episodes using words with different valence or a semantic-related word list. Here, the neutral declarative memory is acquired during a multi-trial training session that allows us to determine the level of learning. Another difference is that in other paradigms, the stressful situation shares the valence with the emotional content of the memory that it is modifying (Payne et al., 2006; Schwabe, Joëls, Roozendaal, Wolf, \& Oitzl, 2012; Schwabe et al., 2008). In our memory task, the stressful situation is not related to the neutral material. This gives us the opportunity to investigate the relationship between threatening situations and neutral memories.

The effects of stress that were administered before acquisition are dissimilar, thereby showing impairment (Kirschbaum, Wolf, May, Wippich, \& Hellhammer, 1996; Lupien et al., 1997; Payne et al., 2006; Payne et al., 2007; Schwabe et al., 2012) or improvements (Nater et al., 2007; Preuß \& Wolf, 2009; Roozendaal, 2000; Schwabe et al., 2008; Smeets et al., 2007; Wolf, 2009). Some reports have shown impairment or no effect on neutral memories, whereas other reports have shown an improvement in short- and long-term memory tests (Payne et al., 2006, 2007; Rimmele, Domes, Mathiak, \& Hautzinger, 2003). Our results agree with the latter. Testing that was performed near training implies that the evaluation might be done when the stress is still elevated, thereby affecting not only the encoding but also the early consolidation and retrieval. In this report, it is possible that either the encoding or the early consolidation improves given that a similar effect emerges two or eight days after training, the time point at which the induced stress vanished.

We adapted the TSST using a virtual auditory panel (VAP) that was generated by only one researcher, who interacted with the 
participants. This design offers an extremely low cost test with an easy protocol. This makes the VAP ideal for large samples because a single researcher administers it, thereby standardizing the interaction with the participants. We demonstrated that the VAP was capable of eliciting subjective stress and SYM axis activation. Moreover, when it was run in parallel with the TSST, a significant increase in salivary cortisol was found showing HPA axis activation. Thus, consistent with theoretical models (Dickerson \& Kemeny, 2004), the social stress response seems to be subject to the relative uncontrollability of task outcome and the presence of social evaluation, both of which are supplied by the VAP.

It is important to highlight the impact of the auditory panel under the VAP in comparison with other versions of virtual committees. It has been proposed that such committees may induce less stress than the panel used in the TSST (Kotlyar et al., 2008). For example, by using specific samples (e.g., adolescents or children), the Leiden Public Speaking Task, which involves speaking in front of a pre-recorded audience, showed a moderate cortisol increase in the adolescent sample (Westenberg et al., 2009). In this report, the VAP showed similar stress responses to the original TSST. To assess the impact of the stress per se, the control situation is created in different ways (Allen et al., 2014; Het, Rohleder, Schoofs, Kirschbaum, \& Wolf, 2009). Here, we designed a control condition with the same phases and similar tasks but without the social interaction (VAPf). Thus, the same physiological measures are registered and are taken as a baseline for comparison with the stressful situation. Based on this comparison, it might be assumed that the VAP induces a stressful situation in spite of the absolute values that were obtained for the different biomarkers.

As in other reports, skin conductance correlated with the level of salivary cortisol and was useful as an indicator of threat response following VAP administration (Buchanan, Tranel, \& Adolphs, 2006; El-Sheikh, Erath, Buckhalt, Granger, \& Mize, 2008; Van Goozen, Matthys, Cohen-Kettenis, Buitelaar, \& van Engeland, 2000). Skin conductance reflects variations in the SYM axis, which are faster than those on the HPA axis (Hermans, Henckens, Joëls, \& Fernández, 2014). This allows us to have an online and reliable measure of the activation of the SYM axis during each step of the procedure; however, due to factors, such as cortisol pulsatility and diurnal patterns (Young, Abelson, \& Lightman, 2004), single time points may show considerable variability. For this reason, multiple measures are necessary to better describe the HPA axis variations. Future experiments with multiple samples would confirm the effectiveness of the VAP in the activation of the HPA axis.

Notably, we found a positive correlation between SCL and cortisol after the administration of the VAP and a positive correlation between SCL and memory in the short-term memory test (Experiments 2 and 3 , respectively). We then predicted that a positive correlation between cortisol levels and memory performance in a short-term memory test would also be expected. Nevertheless, this prediction limits the possibility to distinguish the contribution of the SYM and HPA to memory performance. Future experiments with cortisol and SCL measures could better elucidate their effects on memory.

Stressful events trigger the secretion of catecholamines and glucocorticoids (Schwabe et al., 2012). Fast catecholamine and non-genomic glucocorticoid actions interact in the basolateral amygdale to modify the activity of other brain regions (e.g., prefrontal cortex, hippocampus caudate nucleus), thereby maintaining these regions in a "memory formation mode". Under this mode perception, the attentiveness toward, arousal by, and encoding of different experiences are enhanced. Over time, catecholamines return to their basal level and genomic glucocorticoid actions are initiated, which shift the system to a "memory storage mode". Under this mode, the possibility of encoding new or retrieving old material is impaired, but the long-term storage of the material that was acquired around the stress is improved. Based on the activation of the SYM axis caused by the administration of VAP, our results are in line with the first phase of the model. Thus, the changes in the hippocampus that are associated with this type of memory might explain the strengthening of the neutral declarative memory. Based on our results, we can hypothesize that the SYM and HPA activity could improve during general memory acquisition/retention; however, when the paradigm includes mixed emotional material, the measurements of cortisol levels show a bias toward negative emotional items (Schwabe et al., 2008, 2012; Wolf, 2009).

It is important to highlight the two main contributions of this report. First, despite the difference in emotional valance, we demonstrate that it is possible to modulate a pure neutral memory with a threatening situation. Second, the threat response that was induced by the VAP affects the process of memory encoding. This effect persists, as demonstrated by a long-term memory test, supporting a persistent change in memory strength and preventing forgetting as a consequence of the simple passage of time.

\section{Acknowledgments}

We thank our subjects for their cooperation. We also thank Angel Vidal and Ana Goin for technical assistance, Ero Pesquero and Gonzalo Lopez Brouchoud for software development, Catalina Nemirovsky and Julieta Sztarker for English edition. This work was supported by FonCyT (Grant PICT2006-2261; PICT2010-0391; PICT 2012-0117), by CONICET PIP 2010 No. 11220090100164 and FLENI. The funders had no role in study design, data collection and analysis, decision to publish, or preparation of the manuscript.

\section{Appendix A. Supplementary material}

Supplementary data associated with this article can be found, in the online version, at http://dx.doi.org/10.1016/j.nlm.2015.10.011.

\section{References}

Allen, A. P., Kennedy, P. J., Cryan, J. F., Dinan, T. G., \& Clarke, G. (2014). Biological and psychological markers of stress in humans: Focus on the Trier Social Stress Test. Neuroscience and Biobehavioral Reviews, 38, 94-124.

Benedek, M., \& Kaernbach, C. (2010). A continuous measure of phasic electrodermal activity. Journal of Neuroscience Methods, 190(1), 80-91.

Buchanan, T. W., Tranel, D., \& Adolphs, R. (2006). Impaired memory retrieval correlates with individual differences in cortisol response but not autonomic response. Learning \& Memory, 13(3), 382-387.

Diamond, D. M., Campbell, A. M., Park, C. R., Halonen, J., \& Zoladz, P. R. (2007). The temporal dynamics model of emotional memory processing: A synthesis on the neurobiological basis of stress-induced amnesia, flashbulb and traumatic memories, and the Yerkes-Dodson law. Neural Plasticity, 2007. Retrieved from <http://www.hindawi.com/journals/np/2007/060803/abs/>.

Dickerson, S. S., \& Kemeny, M. E. (2004). Acute stressors and cortisol responses: A theoretical integration and synthesis of laboratory research. Psychological Bulletin, 130(3), 355.

Dongaonkar, B., Hupbach, A., Gomez, R., \& Nadel, L. (2013). Effects of psychosocial stress on episodic memory updating. Psychopharmacology (Berl), 226(4), 769-779.

El-Sheikh, M., Erath, S. A., Buckhalt, J. A., Granger, D. A., \& Mize, J. (2008). Cortisol and children's adjustment: The moderating role of sympathetic nervous system activity. Journal of Abnormal Child Psychology, 36(4), 601-611.

Forcato, C., Burgos, V. L., Argibay, P. F., Molina, V. A., Pedreira, M. E., \& Maldonado, H. (2007). Reconsolidation of declarative memory in humans. Learning and Memory, 14, 295-303.

Forcato, C., Fernandez, R. S., \& Pedreira, M. E. (2013). The role and dynamic of strengthening in the reconsolidation process in a human declarative memory: What decides the fate of recent and older memories? PLOS ONE, 8(4), e61688.

Fritz, C. O., Morris, P. E., \& Richler, J. J. (2012). Effect size estimates: Current use, calculations, and interpretation. Journal of Experimental Psychology: General, 141 (1), 2 .

Hermans, E. J., Henckens, M. J., Joëls, M., \& Fernández, G. (2014). Dynamic adaptation of large-scale brain networks in response to acute stressors. Trends in Neurosciences, 37(6), 304-314. 
Het, S., Rohleder, N., Schoofs, D., Kirschbaum, C., \& Wolf, O. T. (2009). Neuroendocrine and psychometric evaluation of a placebo version of the “Trier Social Stress Test". Psychoneuroendocrinology, 34(7), 1075-1086.

Joëls, M., \& Baram, T. Z. (2009). The neuro-symphony of stress. Nature Reviews Neuroscience, 10(6), 459-466.

Kirschbaum, C., Pirke, K.-M., \& Hellhammer, D. H. (1993). The “Trier Social Stress Test" - A tool for investigating psychobiological stress responses in a laboratory setting. Neuropsychobiology, 28(1-2), 76-81.

Kirschbaum, C., Wolf, O. T., May, M., Wippich, W., \& Hellhammer, D. H. (1996) Stress-and treatment-induced elevations of cortisol levels associated with impaired declarative memory in healthy adults. Life Sciences, 58(17), $1475-1483$.

Kotlyar, M., Donahue, C., Thuras, P., Kushner, M. G., O’Gorman, N., Smith, E. A., \& Adson, D. E. (2008). Physiological response to a speech stressor presented in a virtual reality environment. Psychophysiology, 45(6), 1034-1037.

Lazarus, R. S. (1966). Psychological stress and the coping process. Retrieved from <http://psycnet.apa.org/psycinfo/1966-35050-000>.

Lupien, S. J., Gaudreau, S., Tchiteya, B. M., Maheu, F., Sharma, S., Nair, N. P. V., ... Meaney, M. J. (1997). Stress-induced declarative memory impairment in healthy elderly subjects: Relationship to cortisol reactivity 1. The Journal of Clinical Endocrinology \& Metabolism, 82(7), 2070-2075.

Nater, U. M., Moor, C., Okere, U., Stallkamp, R., Martin, M., Ehlert, U., \& Kliegel, M. (2007) Performance on a declarative memory task is better in high than low cortisol responders to psychosocial stress. Psychoneuroendocrinology, 32(6), 758-763.

Payne, J. D., Jackson, E. D., Hoscheidt, S., Ryan, L., Jacobs, W. J., \& Nadel, L. (2007). Stress administered prior to encoding impairs neutral but enhances emotional long-term episodic memories. Learning E Memory, 14(12), 861-868.

Payne, J., Jackson, E., Ryan, L., Hoscheidt, S., Jacobs, J., \& Nadel, L. (2006). The impact of stress on neutral and emotional aspects of episodic memory. Memory, 14(1), $1-16$.

Preuß, D., \& Wolf, O. T. (2009). Post-learning psychosocial stress enhances consolidation of neutral stimuli. Neurobiology of Learning and Memory, 92(3), 318-326.
Rimmele, U., Domes, G., Mathiak, K., \& Hautzinger, M. (2003). Cortisol has different effects on human memory for emotional and neutral stimuli. NeuroReport, 14 (18), 2485-2488.

Roozendaal, B. (2000). Glucocorticoids and the regulation of memory consolidation. Psychoneuroendocrinology, 25(3), 213-238.

Schwabe, L., Bohringer, A., Chatterjee, M., \& Schachinger, H. (2008). Effects of prelearning stress on memory for neutral, positive and negative words: Different roles of cortisol and autonomic arousal. Neurobiology of Learning and Memory, 90 (1), 44-53.

Schwabe, L., Joëls, M., Roozendaal, B., Wolf, O. T., \& Oitzl, M. S. (2012). Stress effects on memory: An update and integration. Neuroscience and Biobehavioral Reviews, 36(7), 1740-1749.

Smeets, T., Giesbrecht, T., Jelicic, M., \& Merckelbach, H. (2007). Context-dependent enhancement of declarative memory performance following acute psychosocial stress. Biological Psychology, 76(1), 116-123.

Smeets, T., Otgaar, H., Candel, I., \& Wolf, O. T. (2008). True or false? Memory is differentially affected by stress-induced cortisol elevations and sympathetic activity at consolidation and retrieval. Psychoneuroendocrinology, 33(10), $1378-1386$.

Spielberger, C. D., Gorsuch, R. L., \& Lushene, R. E. (1970). Manual for the state-trait anxiety inventory.

Van Goozen, S. H., Matthys, W., Cohen-Kettenis, P. T., Buitelaar, J. K., \& van Engeland, H. (2000). Hypothalamic-pituitary-adrenal axis and autonomic nervous system activity in disruptive children and matched controls. Journal of the American Academy of Child and Adolescent Psychiatry, 39(11), 1438-1445.

Westenberg P. M., Bokhorst C. L. Miers, A. C. Sumter S. R., Kallen, V. L. van Pelt, J, \& Blöte, A. W. (2009). A prepared speech in front of a pre-recorded audience: Subjective, physiological, and neuroendocrine responses to the Leiden Public Speaking Task. Biological Psychology, 82(2), 116-124.

Wolf, O. T. (2009). Stress and memory in humans: Twelve years of progress? Brain Research, 1293, 142-154.

Young, E. A., Abelson, J., \& Lightman, S. L. (2004). Cortisol pulsatility and its role in stress regulation and health. Frontiers in Neuroendocrinology, 25(2), 69-76. 